\title{
Geçmişten Günümüze Kablosuz Mobil İletişim Teknolojileri
}

\author{
Ramazan Akkurt ${ }^{1 *}$ \\ ${ }^{1 *}$ Mersin Üniversitesi, Mühendislik Fakültesi, Bilgisayar Mühendisliği Bölümü, Mersin, Türkiye, (ORCID: 0000-0003-2319-9887), rakkurt@mersin.edu.tr
}

(1st International Conference on Applied Engineering and Natural Sciences ICAENS 2021, November 1-3, 2021)

(DOI: $10.31590 /$ ejosat.991625)

ATIF/REFERENCE: Akkurt, R. (2021). Geçmişten Günümüze Kablosuz Mobil İletişim Teknolojileri. Avrupa Bilim ve Teknoloji Dergisi, (28), 115-119.

Öz

Geçmişten günümüze gelişen ve kullanımı giderek artan mobil cihazlar, bu artışla beraber mobil kablosuz iletişim teknolojilerinin de gelişimine öncülük etmiştir. Bu cihazların en temel özelliği olan taşınabilirlik, her ortamda kesintisiz olarak iletişime devam edebilmeleri için mobil iletişim teknolojilerinin gelişmesinde en büyük etkendir. Yıllar içinde gelişen ve büyük coğrafik alanlarda kablosuz iletişim servisleri sağlayan hücresel ağlar, artan cihaz sayısı ve talep edilen iletişîm yükünü karşılayabilmek için süre gelen yıllarda birçok yeni jenerasyon ( $1 \mathrm{G}, 2 \mathrm{G}, 3 \mathrm{G}, 4 \mathrm{G}$ vb.) ağ teknolojisi ile bu alanda iletişim sağlayan en temel ağlardır. Bu çalışmada yeni ve eski model hücresel ağ teknolojilerinin gelişimi irdelenmiş ve yıllara göre büyüyen ağ şartlarındaki iyileşmeler araştırılmıştır. Ve bu bağlamda, gelecek nesil hücresel ağ teknolojilerinin tahmini ağ kapasiteleri hakkında bazı çalışmalar irdelenmiştir.

Anahtar Kelimeler: Hücresel ağlar, Kablosuz ağlar, 5G, Radyo erişim ağları (RANs), Taşınabilir cihazlar.

\section{Wireless Mobile Communication Technologies from Past to Present}

\begin{abstract}
Mobile devices, which have developed from the past to the now and whose use is increasing, have also led the development of wireless communication technologies. Portability, which is the most basic feature of these devices, is the biggest factor in the development of mobile communication technologies so that these devices can maintain communication in any environment without interruption. Cellular networks, which have developed over the years and provide wireless communication services in large geographical areas, are the most basic networks that provide communication in this area with many new generation $(1 \mathrm{G}, 2 \mathrm{G}, 3 \mathrm{G}, 4 \mathrm{G}$ etc.) network technologies in order to handle the increasing number of devices and the demanded communication load in the coming years. In this paper, the development of new and old model cellular network technologies has been examined and the improvements in network conditions that have grown over the years have been investigated. And in this context, some works on the estimated network capacities of next generation cellular network technologies are examined.
\end{abstract}

Keywords: Cellular networks, Wireless networks, 5G, Radio access networks (RANs), Portable devices.

\footnotetext{
*Sorumlu Yazar: rakkurt@mersin.edu.tr
} 


\section{Giriş}

Günümüz koşullarında en büyük bilgi alışverişi kaynaklarından biri olan mobil iletişim, bu yöndeki eğilimlerin sürekli artması sebebiyle son yıllarda büyük gelişmelerin odağı olmuştur. Cisco Annual Internet Report'a göre 2023 yılı itibari ile 13,1 milyar mobil cihazın kullanılacağı tahmin edilmektedir [1]. Her yıl giderek artan bu sayılar, bu cihazların iletişim yüklerinin de artması ile yeni ağ teknolojilerine ve kaynaklarına olan talebi arttırmaktadır. Hareket sınırlaması olmadan, her yerden iletişime devam etme çabasını taşıyan bu cihazlara, geniş kapsama alanlarında servis sağlayabilmek ve yüksek veri alışveriş oranları sunmak, mobil iletişim teknolojilerindeki temel kaygılardır. $\mathrm{Bu}$ çerçevede gelişen ve gelişmeye devam eden mobil kablosuz iletişim teknolojileri, belirli aralıklarla güncellenen ve ortaya çıkan yeni nesil ağ jenerasyonları ile bu isteklere cevap verebilecek teknolojiler oluşturmayı hedeflemişlerdir.

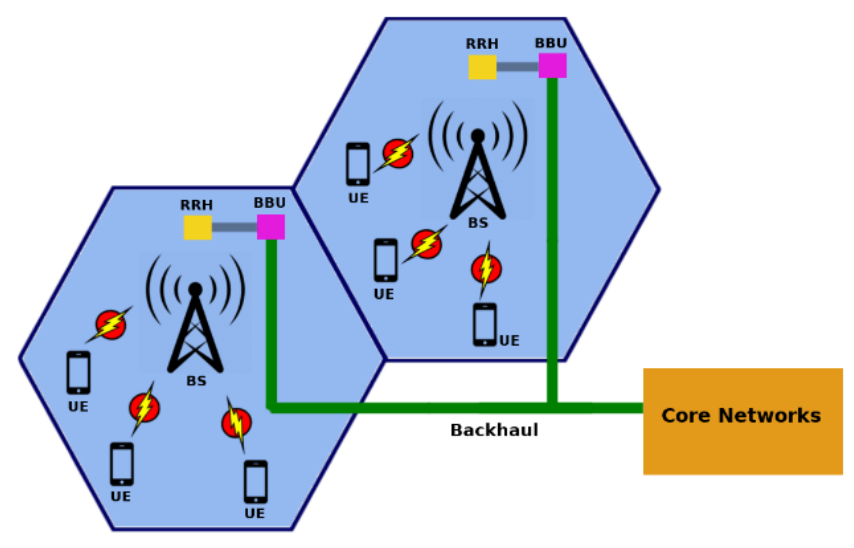

Şekil 1. Hücresel Ăg modeli

Şekil 1'de de gösterilen hücresel ağ modeli, mobil iletişim teknolojilerinde en temel ağlardan biri konumundadır. Bu ağlar, kapasite ve kapsama alanları bakımından çeşitlilik gösteren BS (Base Stations) erişim noktaları sayesinde son kullanıcılarla ilk iletişimi kurarak, herhangi bir konum kısıtlaması olmadan UE'lerin (User equipment) sorunsuz şekilde çekirdek ağlarla (Core networks) iletişim kurmalarını sağlamaktadırlar. Gelişen ağ şartları ve kullanıcı sayısı ile birlikte birçok yeni nesil ağ modellerinin ortaya çıkması kaçınılmaz hale gelmiştir. Bu çalışma, eski ve yeni nesil ağ teknolojileri (1G, 2G, 3G, 4G, 5G vb.) üzerinde genel bir bakış oluşturmayı amaçlamaktadır. Bu ağlarda kullanılan yeni model yaklaşımlar, genel kavramlar, kullanılan yeni teknolojiler ve birçok benzeri özelliklerin ortaya konulması için, bölüm II de bu yeni nesil ağların genel kapsamı ele alınmıştır. Bölüm III'te bu ağların birbirlerine olan üstünlükleri, avantajları ve dezavantajları tablo halinde verilip, genel değerlendirilmesi yapılmıştır. Bölüm IV 'te ise gelecek yıllarda tanitılması beklenen yeni nesil (6G, $7 \mathrm{G}$ vb.) a a modellerinin tahmini ağ kapasite ve özelliklerinin incelenmesi gerçekleştirilmiştir.

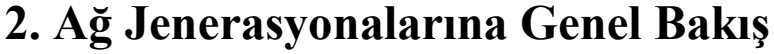

Bu bölümde kullanıcı taleplerine göre gelişen ve değişen yeni nesil ağların genel yapısı, kullanılan yeni teknolojiler ve ağ kaynaklarının genel özellikleri incelenmiştir.

\subsection{G (First Generation)}

1980'li yıllarda ilk olarak tanıtılan $1 \mathrm{G}$ ağ modeli, analog sinyal teknolojisinin kullanıldığı ve yaklaşık $2.4 \mathrm{Kbps}$ iletim hızı destekleyen ilk hücresel ağ jenerasyonudur. Ses verisi dışında başka bir veri paylaşımını desteklememektedir. Advanced Mobile Phone System (AMPS) standardının kullanıldığı bu modelde, farklı bölgelerde Nordic Mobile Telephone (NMT) ve Total Access Communications System (TACS) gibi farklı standartlarda kullanılmıştır. Devre anahtarlama (Circuit switching) teknolojisinin kullanıldığı bu model de hareket halindeki bir kullanıcıya servis sağlanması mümkün değildir [2].

\section{2. $2 \mathrm{G}$ (Second Generation)}

1990'lı y1llarda kullanılan 2G ă̆ modeli, dijital sinyal teknolojisinin kullanıldığı ve yaklaşık $10 \mathrm{Kbps}$ iletim hızlarını destekleyen ikinci nesil ağ teknolojisidir. Global Systems for Mobile communications (GSM) standardının kullanıldığ 1 bu nesil ayrıca Short Message Service (SMS) ve e-mail servislerinin kullanılmaya başlandığı ilk nesil ağ modelidir. Code Division Multiple Access (CDMA) ve Time Division Multiple Access (TDMA) çoğullama teknolojilerini kullanıldığ 1 bu model, ses ve veri iletimini birlikte desteklemektedir [3]. $1 \mathrm{G}$ modelinde olduğu gibi bu modelde de bant genişliğinin kullanıcılar arasında paylaştırılması için devre anahtarlama (Circiut switching) yöntemi kullanılmaktadır. $\mathrm{Bu}$ ağın sonraki yıllarında ara versiyonları $(2.5 \mathrm{G}$ ve $2.75 \mathrm{G})$ olarak tanıtılan bazı yeni nesil alt ağ modelleri de mevcuttur. $2 \mathrm{G}$ ağ jenerasyonun temel teknolojilerini taşıyan 2.5G ara model ağ, General Packet Radio Services (GPRS) erişim teknolojisinin kullanımı ile 50Kbps iletim hızlarını desteklemektedir. Multimedia Messaging Service (MMS) mesajlaşma servisinin ilk kez kullanımı 2.5G ağlarında ortaya çıkmıştır. Diğer bir alt ağ modeli olan $2.75 \mathrm{G}$, Enhanced Data Rate for GSM Evolution (EDGE) teknolojisi ile iletim hızları $144 \mathrm{Kbps}$ 'lara kadar ulaşmaktadır. $1 \mathrm{G}$ ve $2 \mathrm{G}$ den farklı olarak 2G'nin bu alt versiyon iki ağ modelinde paket anahtarlama (Packet switching) kullanılmıştır.

\subsection{G (Third Generation)}

2000 yılının sonlarında geliştirilen 3. Nesil ağ modeli, yaklaşık $384 \mathrm{Kbps}$ iletim hızlarını desteklemektedir. Bir önceki modellere göre ses ve veri iletiminin yanı sıra görüntülü arama özelliğinin ilk kez desteklendiği ağ modeli $3 \mathrm{G}$ ağlarıdır. CDMA2000 gibi yeni model erişim teknolojilerinin yanı sıra, Wideband CDMA (WCDMA) ve Universal Mobile Telecommunications Systems (UMTS) erişim teknolojilerinin de kullanımı 3G ağlarla mümkün olmuştur. $3 \mathrm{G}$ ağları, sonraki yıllarda yeni teknolojilerle donatılmış yeni versiyon (3.5G ve $3.75 \mathrm{G})$ ağ modelleri ile gelişmiş ağ kaynakları sunmaktadır. HSDPA (High-Speed Downlink Packet Access) erişim teknolojisi ile 2Mbps iletim hızlarına ulaşabilen bu yeni versiyon ağ modeli $3.5 \mathrm{G}$ olarak isimlendirilmektedir. HSUPA (High-Speed Uplink Packet Access) erişim teknolojisi ile $30 \mathrm{Mbps}$ gibi iletim hızlarına ulaşmayı başaran bu yeni versiyon $3 \mathrm{G}$ ağı, $3.75 \mathrm{G}$ olarak adlandırılmaktadır [4]. Bazı kaynaklarda HSPA 3.5G, HSPA+ ise 3.75G olarak da adlandırılmaktadır.

\subsection{G (Fourth Generation)}

2010 y1lı itibariyle mobil hücresel ağ teknolojilerinin kullanmaya başladığı $4 \mathrm{G}$ ağ modeli, günümüz itibariyle de çeşitli geliştirilmiş versiyonları ile dünya genelinde aktif olarak kullanılan mobil hücresel ağ jenerasyonudur. İletim hızı olarak 100Mbps gibi hızları destekleyen bu ağda, Long Term Evolution 
(LTE) ve Mobile Worldwide Interoperability for Microwave Access (M-WIMAX) gibi erişim teknolojileri kullanılmaktadır. LTE advanced, LTE advanced pro (4.5G olarak da adlandırılabilmektedir.) ve $4.5 \mathrm{G}+$ gibi gelişmiş ağ versiyonları ile kullanıcı deneyimini oldukça yükselten bu ağlarda, Orthogonal/Single Carrier frequency-division multiplexing (OFDM / SC-OFDM) çoğullama teknolojisi kullanılmaktadır [5]. Veri iletim modelinde ise paket anahtarlama (Packet switching) kullanılmaktadır.

\subsection{G (Fifth Generation)}

2015 yılı itibariyle duyurulan yeni nesil 5G ağları, mevcut tarihlerde tüm lokasyonlarda kullanılmasa da bazı ülkelerde kullanımdadır. Son versiyon olan bu hücresel ağ modeli, WWWW (World Wide Wireless Web) and IPv6 teknolojileri ile donatılmış güçlü bir hücresel yapıya sahiptir. Veri iletim hızı 1Gbps'dan daha yüksektir. Yüksek kapasiteli kapsama alanları ile kullanıcı mobilitesinin sıklığı ve kapsamına bakılmaksızın, büyük coğrafik alanlarda servis sağlayabildiği için yüksek bir kullanıcı deneyimi sunmaktadır. Altyapısında barındırdığ 1 teknolojilerle, 5G ağları oldukça güvenli bir iletişim altyapısına sahiptir [6]. Hem devre anahtarlama (Circiut switching) hem de paket anahtarlama (Packet switching) teknolojilerinin bir arada bulunduğu bu ağlarda, CDMA ve Beam Division Multiple Access (BDMA) çoğullama teknolojisi kullanılmaktadır. Akıllı şehirlerin gelişimi, sağlık hizmetleri için hassas gecikme ve hata toleransı olmayan uygulamalar ve IOT (Internet Of Things) cihazlarının sorunsuz iletişimi için yüksek veri hızları ve bant genişliği sunan bu yeni nesil ağ,

MEC (Mobile Edge Computing) sistemiyle birlikte gecikme yönünden hassas bazı uygulamalar (Augmented Reality, Streaming vb.) için düşük gecikme süreleri ile servis sağlamaktadır [7,8]. MEC sistemi cloud kaynaklarının (Execution, Storage vb.) kullanıcıya daha yakın şekilde konumlandırılmış yaklaşımını temel alan bir teknolojidir [9].
Merkezi cloud sistemleri sabit bir konumda servis sağladıkları için uzun gecikme sürelerine sebep olmaktadır. 5G altyapısı ve sunduğu iletim hızı desteği ile cloud kaynakları, dağıtık bir şekilde $5 \mathrm{G}$ hücresel ağlarının erişim noktalarına konumlandırıldığı bir senaryo son yıllarda ortaya çıkmıştır. Son kullanıcıya yakınlığı ile düşük gecikme süreleri ile servis sağlayan MEC sistemi büyük uygulamaların mobil tarafinda da sorunsuz şekilde kullanılmalarına katkı sağlamaktadır. Ayrıca bu kaynakların kullanımı ile mobil cihazlar enerji tasarrufu ile pil tüketimlerini çok düşük seviyelere çekebilmektedirler. 5G ağları sahip oldukları güçlü ağ kaynakları ile bu sistemlerin sorunsuz şekilde servis sağlamalarında anahtar rol oynamaktadırlar.

\section{3. Özellik ve Performans Karşılaştırması}

Bu bölümde yukarda derlenen hücresel ağ modellerinin genel yapısı tablolar halinde verilerek, yıllara göre değişen ve gelişen ăg şartlarındaki iyileşmeler ortaya konmuştur. Yüksek çözünürlüklü videolar, canlı yayınlar vb. uygulama verilerinin mobil cihazlarda kullanımıyla birlikte bu cihazların ağ kullanımı giderek artmaktadır. $\mathrm{Bu}$ artış mevcut ağların yetersiz kalmasına sebep olmaktadır. Mevcut bir ağ ortamındaki kullanıcıların sorunsuz şekilde iletişime devam etmeleri için var olan ağların iyileştirilmesi kaçınılmaz hale gelmektedir. Artan cihaz ve kullanıcı sayısı ile ağ şartlarındaki iyileşmeler doğru orantılı bir şekilde artış göstermiştir. Yıllar içinde birçok yeni teknoloji geliştirilmiş veya var olan teknolojiler iyileştirilmiştir. Bu kapsamda bu bölümde ağların sunduğu veri iletim hızları, kullandıkları standartlar ve birçok yeni teknoloji derlenmiştir. Yeni teknolojiler bazı eski teknolojilerin yerini almış veya var olan teknolojiler güncel halleriyle daha performanslı hale getirilerek kablosuz ağ teknolojilerine dahil edilmiştir. Belli standartlar yetersiz kaldığı için kullanıcı sayısının daha az olduğu kırsal alanlarda kullanımına devam edilmiştir. Yoğun kullanıcıların bulunduğu alanlarda ise yeni ve gelişmiş teknolojiler kullanılmaya başlanmıştır.

Tablo 1. Ağların Genel Özellikleri

\begin{tabular}{|c|c|c|c|c|c|c|}
\hline Nesiller & Yll & Standartlar & Servisler & $\begin{array}{l}\text { Çoğullama } \\
\text { Teknolojileri }\end{array}$ & Anahtarlama & Iletim Hızı \\
\hline $1 G$ & 1980 & AMPS & Ses & FDMA & Devre & 2.4Kbps \\
\hline $2 G$ & 1990 & GSM & $\begin{array}{l}\text { Ses+Veri+Sms }+ \\
\text { Email }\end{array}$ & CDMA/TDMA & Devre & $10 \mathrm{Kbps}$ \\
\hline $2.5 G$ & 2000 & GPRS & $\begin{array}{l}\text { Ses+Veri+Sms }+ \\
\text { Email }+ \text { Mms }\end{array}$ & CDMA/TDMA & Devre/Paket & $50 \mathrm{Kbps}$ \\
\hline $2.75 G$ & 2003 & EDGE & $\begin{array}{l}\text { Ses+ Veri } \\
+ \text { +Sms+Email } \\
+ \text { Mms }\end{array}$ & CDMA/TDMA & Paket & $144 \mathrm{Kbps}$ \\
\hline $3 G$ & 2001 & UMTS & $\begin{array}{l}\text { Ses+ Verı } \\
\text { +Görüntülü } \\
\text { Arama }\end{array}$ & $\begin{array}{l}\text { CDMA-2000 } \\
\text { WCDMA }\end{array}$ & Devre/Paket & $384 \mathrm{Kbps}$ \\
\hline
\end{tabular}


Tablo 2. Ağların Genel Özellikleri

\begin{tabular}{|c|c|c|c|c|c|c|}
\hline Nesiller & Yll & Standartlar & Servisler & $\begin{array}{l}\text { Çoğullama } \\
\text { Teknolojileri }\end{array}$ & Anahtarlama & Iletim $\mathrm{Hzz}$ \\
\hline $3.5 G$ & 2003 & HSDPA & $\begin{array}{l}\text { Ses+ Veri } \\
\text { +Görüntülü Arama }\end{array}$ & $\begin{array}{l}\text { CDMA-2000 } \\
\text { WCDMA }\end{array}$ & Paket & $2 \mathrm{Mbps}$ \\
\hline $3.75 G$ & 2003 & HSUPA & $\begin{array}{l}\text { Online Oyun } \\
+ \\
\text { Yüksek } \\
\text { Çözünürlükte Tv }\end{array}$ & $\begin{array}{l}\text { CDMA-2000 } \\
\text { WCDMA }\end{array}$ & Paket & 30Mbps \\
\hline $4 G$ & 2010 & $\begin{array}{l}\text { LTE } \\
\text { M-WIWAX }\end{array}$ & $\begin{array}{l}\text { Online Oyun } \\
+ \\
\text { Yüksek Çözünürlük } \\
\text { Tv }\end{array}$ & $\begin{array}{l}\text { OFDM } \\
\text { SC-OFDM }\end{array}$ & Paket & 100Mbps \\
\hline $4.5 G$ & 2014 & LTE-PRO & $\begin{array}{l}\text { Online Oyun } \\
+ \\
\text { Yüksek Çözünürlük } \\
\text { Vıdeo }\end{array}$ & $\begin{array}{l}\text { OFDM } \\
\text { SC-OFDM }\end{array}$ & Paket & $300 \mathrm{Mbps}$ \\
\hline $5 G$ & 2015 & WWWW & $\begin{array}{l}\text { Yüksek } \\
\text { Çözünürlükte Video } \\
+ \\
\text { Sanal Gerçeklik } \\
\text { Uygulamları }\end{array}$ & BDMA & Paket & $1 \mathrm{Gbps}+$ \\
\hline
\end{tabular}

Tablo 1 ve 2'de derlenen genel özellikler hücresel ağlardaki gelişen ve değişen yeni teknolojilerin genel çerçevesini ortaya koymaktadır. Ses iletimi dışında servis sağlamayan $1 \mathrm{G}$ nesil ağdan sanal gerçeklik uygulamalarına kadar destek sunan $5 \mathrm{G}$ ağlarına ulaşan süreçte ağ koşulları ve kaynaklarının gelişimi gelecek nesil ağların da aynı çerçeve de gelişimi için bir ipucu niteliğindedir. Uçlar arası haberleşmede iletim modeli olarak paket ve devre anahtarlama metotları kullanımı dışında yeni bir model kullanılmamıştır. Devre anahtarlama modeli sonraki ağ nesillerinde yerini tamamen paket anahtarlamaya biraktığı görülmektedir. Devre anahtarlama modelinde uç düğümler arasında özel ayrılmış haberleşme yolu oluşturulur. Bağlantı boyunca ayrılan bu yol kurulu kalır ve veri iletişimi bu yol üzerinden gerçekleştirilir. Bağlantı sonlana kadar bu iletişim yoluna başka bir uç düğümün girmesi söz konusu değildir. Paket anahtarlama da ise veri küçük boyutlarda paketlere ayrılarak uçlar arası paylaştırılır. Devre anahtarlamada ki gibi bir veri yolu kurmaya gerek yoktur ve veriler farklı yol kullanarak diğer uç birime ulaşabilirler. Çoğullama teknolojileri mevcut bant genişliğinin o andaki kullanıcılar arasında nasıl paylaştırılacağını belirleyen bir teknolojidir.

\section{Hücresel Ağların Geleceği}

Belli aralıklarda yeni nesil hücresel ağların gelişimi ve kaynak kapasitelerinin artışı, artan cihaz ve kullanıcı sayısı baz alındığında kaçınılmaz hale gelmektedir. Cisco ve benzeri kuruluşların gelecek yıllar için tahmini kullanıcı ve cihaz sayıları baz alınarak, bu sayılara cevap verebilecek kapasitede yeni ağ modelleri geliştirilmeye devam edilecektir. Bu kapsamda son versiyon olan hücresel kablosuz ağ teknolojisi $5 \mathrm{G}$ ile entegre şekilde çalışabilecek veya onun yerini alabilecek $6 \mathrm{G}, 7 \mathrm{G}$ veya $7.5 \mathrm{G}$ gibi temel ağ teknolojilerinin ortaya çıkacağ 1 tahmin edilmektedir. Literatürde gelecek nesil hücresel ağ teknolojilerinin nasıl şekillenmesi gerektiği ve beklenen ağ kaynaklarının özelliklerinin nasıl olması gerektiği konusunda bazı çalışmalar mevcuttur. [10-12]'da yazarlar 6G ve 7G gibi gelecek nesil ağların genel yapısı hakkında ve bu ağlarda mevcut olması beklenen teknolojiler hakkında bazı çıkarımlar yapmışlardır. Yeni nesil $6 \mathrm{G}$ ve $7 \mathrm{G}$ ağ modellerinde son model olan $5 \mathrm{G}$ ağlarından temel fark kapsama alanındaki büyük değişiklikler olarak ortaya konmuştur. $5 \mathrm{G}$ ve öncesi ağ nesillerinde kullanıcıların hizmet aldığı kapsama alanlarına ek olarak global bir kapsama alanı sunmak için uydularla entegre bir kapsama alanı yapısı beklendiği vurgulanmaktadır. Global position system (GPS), COMPASS, Galileo, GLONASS gibi birçok uydu ağının olduğu mevcut 
durumda, bu uydu ağları arasında hand-off ve roaming gibi servis migrasyonlarının sağlanmasında bazı zorluklar ve maliyetler olacağ 1 belirtilmektedir.

Tablo 3. $6 G$ ve $7 G$ Ağlarının Genel Özellikleri

\begin{tabular}{|c|c|c|c|c|}
\hline Nesiller & Yıl & Çoğullama & $\begin{array}{c}\text { Veri } \\
\text { Iletim } \\
\text { Hızı }\end{array}$ & Standartlar \\
\hline $6 G$ & 2030 & CDMA & $11 \mathrm{GBPS}$ & $\begin{array}{c}\text { Global Uydu } \\
\text { Ağları }\end{array}$ \\
\hline $7 G$ & 2030 & CDMA & $11 \mathrm{GBPS}+$ & $\begin{array}{c}\text { Global Uydu } \\
\text { Ağları }\end{array}$ \\
\hline
\end{tabular}

$\mathrm{Bu}$ sorunların yanı sıra tablo 3'de de gösterildiği üzere bu ağların veri iletim hızlarının $11 \mathrm{Gbps}$ ve üstü olacağı ön görülmektedir.

\section{Sonuç}

$\mathrm{Bu}$ çalışmada kablosuz hücresel ağ teknolojilerinin genel çerçevesi ele alınmıştır. Bu kapsamda geçmişten günümüze değişen ve gelişen hücresel ağların ağ kaynaklarındaki gelişmeler, kullanılan teknolojiler ve gelecek yıllarda ortaya çıkması beklenen bazı ă̆ modelleri hakkında bir ön görü sağlaması hedeflenmiştir. Kullanıcı taleplerinin mobil tarafa kayması ve mobil cihazların kullanımının artışı, iletişim kısmında da bu cihazların mobilitesi göz önünde alındığında kablosuz iletişim teknolojilerinin en temel iletişim ağı olarak hücresel ağlara olan ihtiyacı doğrudan arttırmaktadır. Büyüyen cihaz ve kullanıcı havuzu ile hücresel ağların gelişimi sürekli olarak devam edeceği ön görülmektedir.

\section{Kaynakça}

[1] Cisco, U. (2020). "Cisco annual internet report (20182023)",https://www.cisco.com/c/en/us/solutions/collateral/ executive-perspectives/annual-internet-report/white-paperc11-741490.html

[2] Yadav, R. (2017, March). "Challenges and evolution of next generations wireless communication." In Proceedings of the International MultiConference of Engineers and Computer Scientists (Vol. 2).

[3] del Peral-Rosado, J. A., Raulefs, R., López-Salcedo, J. A., \& Seco-Granados, G. (2017). "Survey of cellular mobile radio localization methods: From $1 \mathrm{G}$ to 5G." IEEE Communications Surveys \& Tutorials, 20(2), 1124-1148.

[4] Mondal, S., Sinha, A., \& Routh, J. (2015). A survey on evolution of wireless generations $0 \mathrm{G}$ to $7 \mathrm{G}$. International Journal of Advance Research in Science and Engineering (IJARSE), 1(2), 5-10.

[5] Gupta, A., \& Jha, R. K. (2015). "A survey of 5G network: Architecture and emerging technologies." IEEE access, 3, 1206-1232.

[6] Gohil, A., Modi, H., \& Patel, S. K. (2013, March). 5G technology of mobile communication: A survey. In 2013 international conference on intelligent systems and signal processing (ISSP) (pp. 288-292). IEEE.
[7] Ezhilarasan, E., \& Dinakaran, M. (2017, February). A Review on mobile technologies: 3G, 4G and 5G. In 2017 second international conference on recent trends and challenges in computational models (ICRTCCM) (pp. 369373). IEEE.

[8] Agiwal, M., Roy, A., \& Saxena, N. (2016). Next generation 5G wireless networks: A comprehensive survey. IEEE Communications Surveys \& Tutorials, 18(3), 1617-1655.

[9] Habibi, M. A., Nasimi, M., Han, B., \& Schotten, H. D. (2019). A comprehensive survey of RAN architectures toward 5G mobile communication system. IEEE Access, 7, 70371-70421.

[10] Mihret, E. T., \& Haile, G. (2021). "4G, 5G, 6G, 7G and Future Mobile Technologies.” J Comp Sci Info Technol, 9(2), 75 .

[11] Tidke, R. P., Uttarwar, P. S., Dandwate, D. S., \& Tupe, U. J. (2020). A Literature Review On: Wireless Technologies From $0 \mathrm{G}$ to $7 \mathrm{G}$.

[12] Shoewu, O. O., \& Ayangbekun. (2020). Oluwafemi, J. Insights into the development trends in $7 \mathrm{G}$ mobile wireless networks. 